\title{
Strategies to Enhance Utilization of National Hospital Insurance Fund Scheme Medical Cover by Informal Sector Populations in Kakamega County, Kenya
}

\author{
Mukhwana Eugine Sundays ${ }^{1, *}$, Ngaira J.K ${ }^{2}$, Mutai $C^{3}$ \\ ${ }^{1}$ Masinde Muliro University of Science and Technology, Kenya \\ ${ }^{2}$ Department of Disaster Management and Sustainable Development, Masinde Muliro University of Science and Technology, Kenya \\ ${ }^{3}$ Department of Public Health and Biomedical Sciences, Masinde Muliro University of Science and Technology, Kenya
}

Copyright $(\mathcal{C} 2015$ by authors, all rights reserved. Authors agree that this article remains permanently open access under the terms of the Creative Commons Attribution License 4.0 International License

\begin{abstract}
Social Health Insurance (SHI) has been preferred form of financing health care to reduce out of pocket expenditure on health care. Most countries are extending SHI programmes to people outside the formal sector in an effort to increase access to healthcare. However, this approach faces challenges of enrolment of sufficient numbers of people into a common risk pool and collection of contributions. The objective of this study was to evaluate strategies to enhance participation of people in the informal sector in Kakamega County in the National Hospital Insurance Fund (NHIF) Scheme for access to health care. This was a descriptive qualitative study with sample units purposively selected. Ten Focus Group Discussions were conducted and several Key informants interviewed. Data obtained was scrutinized for emerging themes. The study established that people in the informal sector in Kakamega County were aware of the value of the NHIF cover for accessing health care but lack accurate information on the insurance function of the NHIF cover. Rigid scheme design features also discourage subscription. Major recommendations included policy review of rigid scheme conditions, making the scheme attractive and increasing registration centers. Policy decisions should focus on educating people working outside formal employments on insurance functions of the NHIF cover.
\end{abstract}

Keywords Participation, Access to Health Care, Strategies, Sustainability, National Hospital Insurance Fund Scheme

\section{Introduction}

Access to healthcare (services of providing medical care) is still a global problem because many people cannot afford costs of health services $[20,35]$. Most households still rely on Out-of-pocket payments for health care [3, 28]. Reliance on out-of pocket payments deters people from seeking health care when needed and those who do seek health care face a problem of financial impoverishment [36, 39]. This problem is particularly severe in developing nations where many people live in poverty [34]. Over the last decade, Social Health Insurance (SHI) has emerged as a preferred form of financing of health care costs in most countries [29]. Health insurance mechanisms help people to pool resources and transfer risks of unforeseeable healthcare costs for a pre-determined fixed contribution thereby avoiding catastrophic financial burden. In SHI schemes, people can access health care based on the need and not on ability to pay for health services. Countries with existing National health insurance programs for formal sector workers have recently extended SHI programmes to people outside the formal sector in an effort to increase access to healthcare [38]. The major challenge of adoption of SHI has been integration of the poor and the expanding informal sector that comprises the majority of the workforce into SHI schemes. The bulk of working age adults in developing countries is comprised of the unemployed, subsistence farmers, and those working in the informal sector $[1,33]$.

Social Health Insurance is especially designed for poor and vulnerable people to provide them health and medical facilities since they may not afford the cost of medicine and hospitalization as other regular income people at the time of ill health. A number of studies show that households in the informal sector rely on traditional coping responses such as selling assets and informal borrowing to deal with the adverse consequences of ill-health $[5,32, \& 40]$. These coping responses are not cost free but entail a compromise, protecting current consumption at the cost of future vulnerability [9]. To overcome this problem, the World Health Organization (WHO) recommended social protection in 2005 as a strategy to attain Universal Health Coverage [37]. Since the 2005 World Health Assembly Resolution -WHA R 58.33 [37], policy formulation in most developing countries has embraced SHI as a strategy to 
attain Universal Health Coverage (UHC) because SHI supports the purpose of promoting equity in access to health care. However, developing effective approaches for access to Health care for people in the informal sector through SHI is still a challenge, particularly in low and middle-income countries [2]. Approaches based on health insurance still face challenges of enrolment of a sufficient number of people into a common risk pool and in collection of contributions.

In Kenya, the National Hospital Insurance Fund (NHIF) scheme is still a more accessible medical cover offering insurance at costs that are considerably below the actuarially fair price suitable for most socioeconomic groups in the country [7]. Private health insurance is available but predominantly accessible to the middle and higher-income groups, while, the Community Based Health Insurance (CBHI) schemes still have limited coverage countrywide [23, 22]. The benefits of NHIF membership include coverage of inpatient expenses (costs of bed, meals, treatment and drugs) with the share of expenses covered determined largely by the type of health facilities (Hospitals, Nursing Homes and Dispensaries). Beneficiaries of the scheme also include the contributor's dependents (the spouse and children less than 18 years of age). People in the informal sector above the age of eighteen years can join the scheme voluntarily by paying monthly contributions of Kshs.160 (about US \$2). Although the scheme is open to all since 2011, there appears to be resistance from the informal sector and the unemployed in joining the scheme. Poor participation by people in the informal sector in scheme has been blamed on the difficulties they face. People in the informal sector face difficulties of low and irregular incomes; this makes them unable to make timely contributions [18]. People in the informal sector also face difficulties created by inflexible scheme design features such as penalties, inflexible payment schedules, non-portability of scheme services, cumbersome enrolment and contribution procedures [27]. Poor participation weakens service delivery by the scheme is because the principle of risk pooling requires sufficient membership and regular contributions to pay for health services. It is therefore necessary to establish mechanisms or approaches that can be applied to improve informal sector participation to make the NHIF scheme sustainable.

The purpose of this study was to evaluate strategies to enhance participation of people in the informal sector in Kakamega County in the National Hospital Insurance Fund (NHIF) Scheme. Kakamega County is the second most densely populated County in Kenya with majority of residents deriving livelihoods through the informal sector $([17,15]$. People in the informal sector often work in poor, sub-standard working conditions and are exposed to various hazards without proper knowledge concerning the use of personal protective equipment, and stand a higher risks of injuries. These are the people who should be targeted to benefit from the scheme program since unlike the very poor, they have some income though low and irregular. However, little is known about social health scheme patterns of the informal sector populations in Kakamega County. The success of a service delivery program such as the NHIF largely depends on the utilization of services and the satisfaction of the users. Thus, many people in the informal sector should participate by enrolling and making regular contributions to the scheme. Maximizing participation in the NHIF scheme has rippling effects of getting the citizenry (especially the poor and people in the informal sector) by reducing out of pocket expenditure and increasing access to legitimate healthcare, thereby helping to improve their quality of life.

\section{Methods}

This was a descriptive qualitative study utilizing an evaluation study design approach. Evaluation design was considered appropriate since it is useful in assessing the effects of social and development interventions in the real world; these, generally, involve assessment of existing needs and adequacy of existing services (such the NHIF scheme in this case) with a view planning different stages and methods to reach goal of an intervention $([19,4]$. The approach was used to establish, focus on vital facts about people and their opinions so as to provide information on which to base suggestions for service delivery and utilization of the NHIF scheme. Key issues discussed revolved around factors that influence participation of the informal sector populations of Kakamega County in the NHIF Scheme and strategies that could be adopted to enhance participation in the Scheme. Purposive sampling techniques were employed in selecting the respondents. Purposive sampling was preferred in this study so as to attain a representative sample population. In this sampling strategy, a researcher is more likely to get the opinions of the target population, and also likely to overweight subgroups in the study population that are readily accessible [4]. Ten (10) Focus Groups Discussions (consisting of 8-10 members) were conducted with members of various informal sector engagements (Mechanics, Hawkers, Artisans, Bus drivers, Motor cycle riders, shopkeepers, retail traders, Farmers, House helps) for two-hour discussions captured through group discussion and worksheet analysis. Key informant interview guides were also used to collect data. Prior to actual data collection, the instruments pre-tested with the help of four trained research assistants during the month of March, 2015. The qualitative data took an exploratory or conceptual content analysis process which was more ideal. Interpretation was used to give meanings and explain phenomena such as attitudes, perceptions and willingness to enroll and participate in the NHIF scheme. Spearman's rank order correlation was used to analyze data on strategies to enhance enrolment of people in the informal sector of Kakamega County into NHIF Scheme based on ranking by FGDs and Key informants. The research protocol had approval of the National Commission of Science Technology and Innovation (NACOSTI). A summary of the sampling strategies adopted in the study is presented in Table 1 below. 
Table 1. A summary of sampling strategies adopted in the study

\begin{tabular}{ccc}
\hline Study Population Unit. & Sampling method & Sample size. \\
\hline Sub County Administrators. & Purposive. & 4 \\
NHIF regional Management officers. & Purposive. & 3 \\
Hospital Administrators & Purposive. & 4 \\
Managers with other insurance firms in the County. & Purposive. & 4 \\
Managers Small and Medium Enterprise schemes. & Purposive. & 4 \\
Community health workers. & Purposive. & 5 \\
Local opinion leaders. & Purposive. & 10 \\
Managers of NGO's in health sector. & Purposive. & 5 \\
Manager with the Kenya Red Cross, & Purposive. & 1 \\
Patients in Health Facilities & Quota. & 20 \\
FGDs (various informal sector groups). & Quota. & 10 groups of \\
\hline
\end{tabular}

\section{Study Findings}

Data analyses revealed six overarching themes across the ten focus group types and Key informant interviews. The themes identified difficulties (challenges) to participation in the NHIF scheme and proposed strategies to overcome the difficulties. The themes include difficulties faced in NHIF enrolment and contribution, unavailability of the NHIF scheme or alternative schemes; rigid scheme design features (attractiveness of the scheme, level of copayments, customer orientedness, penalties, waivers and exemption; adequate payment modes such as frequency, timing, place of collection, flexibility) and the effectiveness of outreach strategies to improve implementation of NHIF scheme mandate and to expand enrolment.

Many participants acknowledged that participation in SHI had the potential to reduce out of pocket expenditure and increase access to legitimate healthcare, thereby helping to improve their quality of life. There was widespread agreement in all groups that participation the NHIF scheme is, in fact, important for access to health services, regardless of the difficulties (challenges) they face in participation. The appropriateness of the NHIF scheme was especially strong among people in the transport sector (Bus drivers and Motor cycle riders popularly known as 'Boda Boda') participants, where there was a sense of risk in their daily business, in fact, long overdue, especially because there are frequent Road Traffic Accidents (RTA) in the Study area. Interviews demonstrated that majority of the Key informants are able to contrast the benefits and difficulties of participating in the NHIF scheme easily. The difficulties are both sophisticated and complex in-cooperating positive and negative notions about adopting Health Insurance and social consequences. As one key informant remarked:

'I would never participate in a Harrambe (Fund raising) to pay for a hospital bill for a Businessman or Businesswoman because these are people who I expect to be participating in the NHIF scheme as a strategy of preparedness against emergency health care costs'.
Thus, Key informants could not understand why eligible and 'able' persons in the informal sector did not participate in the NHIF scheme and would rather see them suffer for their 'mistake' of non-participation.

Participants in most groups reacted well to messages in materials regarding benefits of participation in the NHIF scheme, however, they expressed concern over perceived government inaction on the absence of essential drugs in health facilities in the past. Participants in the transport industry, in particular, were skeptical about government plans to increase NHIF contributions for better services. They wanted indications of more action in terms of facilities and quality services. A majority of the Key informants acknowledged that participation in the NHIF scheme had the potential of reducing catastrophic Out of Pocket health care expenditure. However, eight of the twenty patients interviewed said using the NHIF medical scheme cover did not make any difference on health care expenditure. One patient remarked:

'The costs of travel form my home to this health facility and the fact that I still have to purchase drugs yet I contribute to the NHIF scheme means that participation in the NHIF scheme is not helpful'. [Male patient aged 35 at the referral hospital]

This finding reveals that there is still some dissatisfaction of NHIF support in meeting health care expenditure. The same observation was made when the issue of the actual time spent at the health facilities was sought for both insured and uninsured. Insured outpatients complained that they spent more time at the facilities than the uninsured when they sought health services. It was also evident that some of the patients who participate in the NHIF scheme neither bothered to use their NHIF cards nor sought re-imbursement for health care expenditure. Moreover, even the Key Informants who were aware of the provision to seek re imbursement, complained of delays and bureaucracies in getting refunds of money spent on health care expenditure. Only 4 out of the 24 key informants interviewed had ever applied for re-imbursement of medical expenditure incurred. 
The concept of sustainable strategies for participation in the NHIF scheme medical cover for access to healthcare is a positive and acceptable one for most participants, regardless of their level of understanding or familiarity with the term. It did, however, raise questions for some who felt that scheme also needs to provide more qualitative and quantitatively clear set of services. Participants in all the Focus Group Discussions (FGDs) expressed a concern over the ability for the NHIF scheme to provide quality services within its mandate, as well as to achieve a long-term outcome of improved health care services. It was interesting to note that when references were made to spouse and children under the age of 18 years (information provided in the NHIF Brochure) being beneficiaries of the scheme, participants appeared reassured. This suggested that participants did not necessarily want to review all the detail, but wanted to know the scheme was readily accessible for assistance in times of health care need.

Participants in all groups had difficulty thinking beyond government subsidies when considering strategies to enhance participation in the NHIF scheme. There were very few mentions of contributions through Savings, Credit and Cooperative Societies (SACCOs) currently under the consideration by NHIF management. When prompted, groups felt that use of SACCOs represent borrowing loans and may cause loss of assets to auctioneers by defaulters due to irregular incomes.

The pattern on suggested strategies to enhance participation in the NHIF scheme across the FGDs and the Key informant interviews point at overcoming the rigid scheme design features which appeared to be major obstacles to participation. Participants were of the opinion that access to health care using the NHIF medical scheme cover could be improved by making enrolment and monthly contribution procedures easier. There are only two main offices in Kakamega town and Mumias (almost centrally placed in the county) town where one can register for membership in the NHIF scheme. Travel to these registration centers involves cost and could be expensive for people like subsistence farmers who live far away in the rural parts of Kakamega County.

Moreover, the procedures of enrolment and contribution are cumbersome; to register, one needs a passport photographs and information about the spouse and birthdays of the children under the age of 18 years which most male participants could not remember accurately. The procedure of contribution involves monthly deposits of Ksh.160 through a given Bank account or through the Mpesa (Electronic Money Transfer System). After making the contribution, one is expected to upload the details in a cybercafés. Participants suggested that the NHIF scheme should set up more registration centers. Across the groups, the general feeling was that the NHIF scheme should have more attractive contribution rates and level of copayments (customer oriented). This can be done by availability of the NHIF scheme services in all health facilities and also the use of mobile registration centers across the county.
Participants also suggested removal of rigid scheme features (barriers) such as harsh penalties and the condition of presentation of birth certificates for children under the age of eighteen years before these young dependents could benefit from the NHIF medical cover. The NHIF scheme regulations are rigid such that any member who fails to make contributions on time (usually by the $5^{\text {th }}$ of the subsequent month) will pay a penalty of five times the usual contribution (i.e. Kshs.800 instead of Ksh.160) to be readmitted and guaranteed of benefiting from the scheme. The NHIF scheme management argued that this condition was meant to discourage default in contributions to the scheme. However, the participants were particularly unhappy about penalties; as one FGD summarized their disapproval:

'With the kind of low and irregular incomes we get in our activities, why would one risk to subscribe to the NHIF scheme when it is apparent that you are not sure to have Ksh.800 for penalty fee if you fail to make contribution by the 5th of the subsequent month?' [One FGD summary note]

Clearly, participants see the scheme condition of penalties as punitive and suggested that this condition should be reviewed. The NHIF scheme management also acknowledged that penalties could discourage potential members. In addition, most contributors appeared unaware of the condition of presenting a birth certificate before the NHIF medical scheme cover could be used to offset medical costs for children under the age of eighteen years. This condition was also seen as punitive by participants. However, the scheme management argued that presentation of birth certificates was only meant to avoid cases of impersonation; there was need to scrutinize and establish legitimate young dependents of the scheme through the use of birth certificates.

Participants were of the view that NHIF scheme outreach strategies to improve implementation of NHIF scheme services and to expand enrolment were poor. Most participants had poor information about the insurance function roles and working of the NHIF scheme. Almost all the key informants observed that the NHIF scheme had suffered negative publicity as a result of media reports of frauds and mismanagement of funds contributed by members. Most participants were not aware of the actual place to register in the NHIF scheme. Similarly, most participants had a misconception that the NHIF medical cover can be used only to cater for inpatient services. 12 of the 20 patients interviewed said they had only presented their NHIF cards when they had serious illness that required hospitalization. In addition, the patients appeared confused about medical insurance cover function as opposed to the general life insurance policy functions. A similar observation was made across all the FGDs and for most key informants. One Key informant argued:

'Why is the NHIF scheme called an insurance scheme yet we never see any bonus being paid to contributors as with other insurance policies?' [Key informant aged 54] 
Clearly, participants lack accurate information about the function of the NHIF medical cover in risk pooling at fair actuarial prices as opposed to profit making private health insurance companies. Managers with other insurance firms explained that their programs succeeded because contributions to their medical schemes were calculated at actuarial prices that sustain the risk pool. The managers admitted that have very few people from the informal sector who participate in the private health insurance schemes. However, Key informants had another suggestion on contributions; they suggested that contributions to the scheme should not be a lifelong event; it should end when one attains a certain age, preferably 55 years. Their argument was that after the age of 55, members had been contributing for long supporting dependents and should therefore be supported by the active working age groups. This would make the NHIF scheme more attractive.

\section{Discussion}

The study established that the NHIF scheme was perceived by participants as beneficial in promoting access to health care for people in the informal sector. Both the insured and uninsured respondents appeared to agree on the usefulness of the NHIF scheme in accessing health care. Findings from the FGDs revealed that a majority of the people in the informal sector are willing to use the NHIF scheme to access health services. However, participation in the scheme appears to be hampered by rigid scheme design features, poor knowledge about the insurance function of the NHIF medical cover, poor image of the scheme and poor outreach strategies by the scheme. The observed poor participation in the NHIF scheme can be explained by unmet health care needs for NHIF members, especially when members of the NHIF scheme still have high out of pocket expenditure like costs of travel to accredited health facilities, buying drugs like nonmembers of the scheme and inflexible scheme design features such as strict datelines to remit contributions. This finding corroborates with findings of a similar study findings by Kimani et al [18] in which he reported that NHIF scheme design features were an impediment to enrolment by people living in the slums of Nairobi, Kenya.

Another key finding was on lack of attractiveness of the scheme and the inability of the scheme to offer qualitative clear set of services. There was a gap between what the NHIF scheme proclaimed to offer in terms of benefits to members and what was actually available. Such issues as lack of essential drugs at the health facilities, lack of certain services at certain facilities and dissatisfaction by the participating members make the NHIF scheme unattractive to potential members in the informal sector. The delays in reimbursement and lack of information on re imbursement might be one of the underlying reasons for the poor attitude towards the scheme. However, the lack of drugs and lack of certain essential services at certain health facilities cannot solely be blamed on the NHIF management. The scheme requires sufficient numbers of contributing participants in the risk pool to raise adequate funds to pay for quality health services. There is need for more accountability of the funds contributed by members to redeem the credibility of the NHIF scheme and make it attractive to potential members from the informal sector. Even then, the scheme could strive to make members feel the privilege of membership by reducing patients waiting time before being attended to.

Poor knowledge on the insurance function of the medical cover could also have affected a sizable proportion of potential members to the NHIF scheme. Poor knowledge on the insurance functions of SHI schemes has been shown to have negative effects on enrolment into the schemes [29]. Where key informants think they should have bonuses as in other insurance policies shows they do not understand the principles behind risk pooling in the NHIF scheme.

\section{Conclusions}

The study set out to evaluate strategies to increase participation in the NHIF scheme. This was achieved by assessing the views of Health care providers, insurance managers, and insured and uninsured clients. The perceived negative attitude of the NHIF scheme was as a result of poor marketing strategies, limited information on the insurance functions of the NHIF medical scheme as well as the negative publicity on the poor management of NHIF contributed funds in media. Besides, the scheme design features were seen by most participants as an obstacle to participation. The NHIF scheme was seen by all participants of the study to be beneficial. The insured were less satisfied with the care given them, according to the study. There is urgent need to address these issues in order to promote confidence in the NHIF, as well as its sustainability for the achievement of universal health insurance coverage.

\section{REFERENCES}

[1] Acharya, A. \& Vellakal, S. (2013). The Impact of Health Insurance Schemes for the Informal Sector in Low- and Middle-Income Countries: A Systematic Review. The World Bank Research Observer.

[2] Acharya A, Vellakkal S, Kalita S, Taylor F, Satija A, Burke M, Masset E, Tharyan P, Ebrahim S. (2014). Do Social health insurance schemes in developing country settings improve health outcomes and reduce the impoverishing effect of healthcare payments for the poorest people? Cochrane Database of Systematic Reviews, 2014.

[3] Chankova S, Atim C, Hatt L. (2010). Ghana's National Health Insurance Scheme; In: Escobar M, Griffi $n$ C, Shaw P, Eds. The impact of health insurance in low-and middle-income countries. Washington, DC: Brookings Institution Press, 2010: 51-88.

[4] Creswell, J.W. (2013). Qualitative inquiry and research 
design; Choosing among the five approaches ( $3^{\text {rd }}$ ed.), Thousand Oaks, CA: sage

[5] Dekker, M. \& Wilms, A. (2010). Health insurance and other risk-coping strategies in Uganda: the case of micro-care insurance. Ltd. World Dev 2010, 38(3):369-378.

[6] Delloite (2011). NHIF strategic review \& market assessment of pre-paid health schemes: measuring up. Nairobi: Delloite, 2011.

[7] Deolitte, C. L. (2011). A Strategic Review of NHIF and Market Assessment of Private Prepaid Health Schemes, Nairobi: Ministry of Medical Services.

[8] Finkelstein, A. and McKnight, R. (2008). What Did Medicare Do? The Initial Impact of Medicare on Mortality and Out of Pocket Medical Spending. Journal of Public Economics, 92(7): 1644-1669

[9] Flores, G., Krishnakumar, J., O'Donnell, O., and van Doorslaer E. (2008). "Coping with Health-Care Costs: Implications for the Measurement of Catastrophic Expenditures and Poverty" Health Economics 17(12): $1393-1412$

[10] Government of Kenya GOK, (2012). Kenya: Facts and figures 2012. Nairobi: Kenya National Bureau of Statistics.

[11] Government of Kenya (GOK), (2004). Sessional Paper on National Social Health Insurance in Kenya. Nairobi: Ministry of Health.

[12] Government of Kenya GOK, (2012). Health Sector Working Group Report Medium Term Expenditure Framework (MTEF) for the period 2012/13-2014

[13] Kenya National Bureau of Statistics (2010): Population and Housing Census Highlights; Nairobi Kenya.

[14] Kenya Demographic and Health Survey-KDHS (2008-2009). Preliminary Report.

[15] Kenya County profiles- KCP (2011); Revenue allocation Authority, Nairobi Kenya.

[16] Kakamega County Data Sheets-KCDS (2012). Revenue allocation Authority, Nairobi Kenya.

[17] Kenya National Bureau of Statistics -KNBS, (2010). Population and Housing Census Highlights; Nairobi, Kenya.

[18] Kimani, J. K, Remare, E., Kyobutungi, C., Mberu, B. and Muindi, K., (2012). Determinants for participation in a public health insurance program among residents of urban slums in Nairobi, Kenya: results from a cross sectional survey; BMC Health Services Research 2012, 12:66.

[19] Kothari, D. (2008). Research Methods simplified. Sage publishers, Bombay, second Edition; P57-89.

[20] Leatherman S, \& Dunford C., (2010). Linking health to microfinance to reduce poverty; Bull World Health Organnisation 2010, 88:470-47.

[21] Manning, W,G.,and Marquis M, S (1996). Health Insurance: The trade-off Between Risk Pooling and Moral Hazard. Journal Economic Perspectives 5

[22] Mathauer I, Schmidt J.O, Wenyaa, M. (2008). Extending social health insurance to the informal sector in Kenya. An assessment of factors affecting demand; International Journal of Heath planning and Management 2008, 23:51-68.

[23] Midiwo, G., (2007). Quality management actors and instruments and their institutional links to social health Protection mechanisms. In Conference on Assuring Quality Health Care through Social Health Protection: The role of strategic purchasing and quality management: 2007.

[24] National Health Insurance Authority- Ghana (2010). National Health Insurance Scheme 2010 annual report. Accra: National Health Insurance Authority, 2011.

[25] National Hospital Insurance Fund Strategic Plan, 2006-2011 (2010). NHIF, Nairobi.

[26] Nyagero J. (2012). 'Predictors for health insurance coverage amongst the older population in Kisii County, Kenya'; A Ph.D thesis in Public Health, Institute of Tropical Medicine and Infectious Diseases, of Jomo Kenyatta University of Agriculture and Technology; Nairobi, Kenya:

[27] Nyagero. J., Gakure, R., Keraka M., Mwangi M. and Wanzala, P. (2012). The background, social support and behavioral characteristics associated with health insurance coverage among the older population in Kisii County, Kenya; Africa Journal of Health Sciences; 2012; 22:201-213.

[28] Osei-Akoto, I.\& Adamba. (2011).Ethnic and Religious Diversity as Determinants of Health Insurance Uptake in Ghana. Institute of Statistical, Social and Economic Research, University of Ghana,Legon Accra.

[29] Panda P, Dror I, Koehlmoos T, Hossain S, John D, Khan J, Dror D. (2013). What factors affect take up of voluntary and community based health insurance programmes in low- and middle- income countries? A systematic review (Protocol). London: EPPI-Centre, Social Science Research Unit, Institute of Education, University of London

[30] Seddoh, A, and Akor S. (2012). Policy initiation and political levers in health policy: lessons from Ghana's health insurance. BMC Public Health 2012; 12 (suppl 1): S10.

[31] Smith, A.,Chamberlain,D.,Hawan,S.,Narb,S.,\& Chelwa,G.(2010). Kenya Micro insurance Landscape: Market and Regulation. The Centre for Financial Regulation and Inclusion.

[32] Sparrow, R., van de Poel, E., Hadiwidjaja, G., Yumna, A., Warda, N. and Suryahadi, A. (2014). "Coping with the Economic Consequences of Ill Health in Indonesia." Health Economics 23(6): 719-728

[33] Valodia, I. and R. Devey (2010). "Formal-informal economy linkages: What implications for poverty in South Africa?" Law, Democracy \& Development 14.

[34] Wagstaff, A (2010). Estimating health insurance impacts under unobserved heterogeneity: the case of Vietnam's health care fund for the poor. Health Economics, 19(2): 189-208.

[35] Wagstaff,, A. (2012). Beyond universal coverage part III http://blogs-worldbank.org/developmenttalk/beyond-univers al-coverage-part-iii. (Accessed on April 30, 2012).

[36] World Health Organization- WHO (2010). The World Health Report- Health systems financing: the path to universal coverage. Geneva: World Health Organization, 2010.

[37] World Health Organization- WHO, (2005). Social health insurance: Sustainable health financing, universal coverage and social health insurance. 58th World Health Assembly 
World Health Organization.

[38] World Health Organization (2010). Paying for health services; Geneva, Switzerland; 2010 at

http://www.who.int/mediacentre/factsheets/fs320.pdf. (Accessed on 2/2/2013).

[39] World Health Organization- WHO, (2010). The World Health Report Health systems financing: the path to universal coverage. Geneva: World Health Organization, 2010. WHO. (2010). "The world health report - Health systems financing: the path to universal coverage". WHO. Fact sheets on paying for healthcare services.

[40] .Yilma, Z., Mebratie, A.D., Sparrow, R.A., Abebaw, D., Dekker, M, Alemu, G and Bedi, A.S. (2014) "Coping with shocks in rural Ethiopia". Forthcoming in Journal of Development Studies 